\title{
Massive particle production from accelerated sources in high magnetic fields
}

\author{
Douglas Fregolente* \\ Universidade Federal de Campina Grande, Centro de Formação de Professores, Cajazeiras, PB, \\ Brazil \\ E-mail: douglasfregolentedcfp.ufcg.edu.br
}

\author{
Alberto Saa \\ Departamento de Matemática Aplicada, UNICAMP, Campinas, SP, Brazil. \\ E-mail: asaa@ime.unicamp.br
}

\begin{abstract}
Non-electromagnetic emissions from high energy particles in extreme environments has been studied in the literature by using several variations of the semi-classical formalism. The detailed mechanisms behind such emissions are of great astrophysical interest since they can alter appreciably the associated energy loss rates. Here, we review the role played by the source proper acceleration $a$ in the particle production process. The acceleration $a$ determines the typical scale characterizing the particle production and, moreover, if the massive particle production is inertially forbidden, it will be strongly suppressed for $a$ below a certain threshold. In particular, we show that, for the case of accelerated protons in typical pulsar magnetospheres, the corresponding accelerations $a$ are far below the pion production threshold.
\end{abstract}

4th School on Cosmic Rays and Astrophysics,

August 25-September 04, 2010

Sao Paulo Brazil

${ }^{*}$ Speaker. 


\section{Introduction}

Processes involving particle production in the presence of classical electromagnetic fields $F_{a b}$ can be considered in the context of semi-classical approximation, in which the source particle is described by a classical current with a prescribed trajectory and the emitted particles are considered as fully quantized fields. For the case of accelerated protons, the inertially forbidden decays via the strong interaction channel like, for instance, $p^{+} \stackrel{a}{\rightarrow} p^{+} \pi^{0}$ and $p \stackrel{a}{\rightarrow} n \pi^{+}$, and via the weak channel, as $p^{+} \stackrel{a}{\rightarrow} n e^{+} v$, have been studied in detail in the semi-classical approximation. For the case of motion under magnetic fields, the validity of the semi-classical approximation is warranted if $[1,2,3]$,

$$
\chi=\frac{B^{\star}}{B_{\text {cr }}} \ll 1,
$$

where $B^{\star}=\gamma B$ is the magnitude of the magnetic field measured in the instantaneous reference frame of the proton, $\gamma$ is the usual Lorentz factor, and $B_{\mathrm{cr}}=m_{p}^{2} / e \approx 1.5 \times 10^{20} \mathrm{G}$ is a critical value for the magnetic field, denoting the region where a full quantized analysis is mandatory. Provided that condition (1.1) is obeyed, the semi-classical calculations are accurate and the associated emitted power depends only on the proper acceleration of the source [4]. For the case of a magnetic field $B$, for instance, the proper acceleration of a (relativistic) proton is $a \approx \gamma e B / m_{p}$ and, consequently, Eq. (1.1) can be rewritten as

$$
\chi=\frac{a}{m_{p}} \ll 1 .
$$

The parameter $\chi$ can be defined in a frame independent way as $\chi=\sqrt{\left(e F^{a b} p_{a}\right)^{2}} / m_{p}^{3}$, where $p_{a}$ stands for the proton momentum. For the case of inertially forbidden particle production, as those ones explicitly listed above, one naturally expects a strong suppression for quasi-inertiall $(\chi \approx 0)$ protons.

We review here the role played by the proper acceleration $a$ of the source in the massive particle production, with emphasis on the ranges favoring the massive particle emission channels. We also discuss the possibility of observing signals of curvature pion radiation from accelerated protons in the magnetosphere of strongly magnetized pulsars. Unless otherwise stated, natural units where $c=\hbar=1$ and the spacetime signature $(+,-,-,-)$ are adopted through this work.

\section{The Semi-Classical Formalism}

We consider here the following kind of decay process

$$
p_{1} \stackrel{a}{\rightarrow} p_{2}+\sum_{i} q_{i}
$$

where $p_{1}$ and $p_{2}$ are the source particles, described by a classical current corresponding to the states of a two level system following a prescribed classical trajectory with proper acceleration $a$, and $q_{i}$ are the emitted particles, described by quantized fields. (For further details, see [5, 6], for instance.) We assume that the respective masses $m_{q_{i}}$ of the emitted particles $q_{i}$ obeys $m_{q_{i}}<m_{p_{1,2}}$, where $m_{p_{1}}$ and $m_{p_{2}}$ stands for, respectively, the masses of the source particles $p_{1}$ and $p_{2}$. If $m_{p_{1}}<m_{p_{2}}+\sum_{i} m_{q_{i}}$, the process is known to be forbidden for inertial trajectories $(a=0)$. It is intuitive to expect that, if the source is supposed to follow a prescribed trajectory, the momentum $\mathbf{k}_{i}$ of the emitted particle 


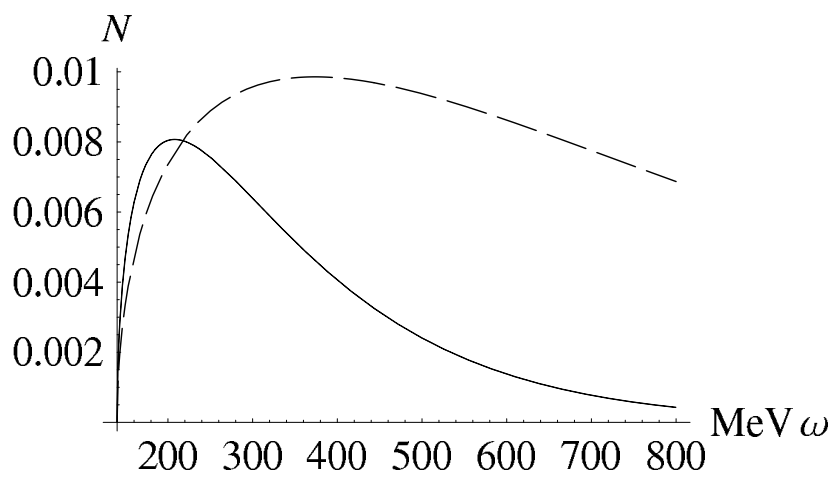

Figure 1: Normalized energy distribution of the emitted scalar meson $\pi^{0}$ field for different values of the proper acceleration of the proton. Full line: $a=150 \mathrm{MeV}$, dashed line: $\mathrm{a}=500 \mathrm{MeV}$.

$q_{i}$, measured in the rest frame of the source, must be constrained to $\left|\mathbf{k}_{i}\right| \ll m_{1,2}[5,6]$. Moreover, the mean energy $\tilde{\omega}_{i}$ of the emitted particles (also measured in the proton's reference frame) is of the order of the source proper acceleration[5], i.e.,

$$
\tilde{\omega}_{i} \sim a
$$

Then, from the condition $m_{q_{i}} \ll m_{1,2}$ and $\tilde{\omega}_{i}^{2}=\left|\mathbf{k}_{i}\right|^{2}+m_{q_{1}}^{2}$ one has $a \ll m_{1,2}$. In order to clarify these points, let us consider the explicit example of the emission of neutral pions by uniformly accelerated protons,

$$
p^{+} \stackrel{a}{\rightarrow} p^{+} \pi^{0}
$$

Without loss of generality, let us assume a uniformly accelerated trajectory along the $z$ direction with worldline given by

$$
x^{\mu}=a^{-1}(\sinh (a \tau), 0,0, \cosh (a \tau)) .
$$

Following the same procedure employed in the Refs. [5, 6], one obtains the differential decay rate as function of the energy of the emitted pion

$$
\frac{d \Gamma}{d \tilde{\omega}}=\frac{G_{\mathrm{eff}}^{2}}{2 \pi^{2} a} \sqrt{\tilde{\omega}^{2}-m_{\pi}^{2}} K_{0}(\tilde{\omega} / a),
$$

where $K_{0}$ is the modified Bessel function of order $0, m_{\pi} \approx 140 \mathrm{MeV}$ is the $\pi^{0}$ mass, and $G_{\text {eff }}$ is the effective coupling constant. The total emitted power is

$$
W=\frac{G_{\mathrm{eff}}^{2} m_{\pi}^{3}}{\pi^{5 / 2} a} G_{13}^{30}\left(\frac{m_{\pi}^{2}}{4 a^{2}} \mid \begin{array}{c}
0 \\
-1 / 2,0,0
\end{array}\right),
$$

where $G_{m n}^{p q}$ stands for the Meijer G-function[7]. The corresponding normalized energy distribution,

$$
N=\frac{1}{\Gamma} \frac{d \Gamma}{d \tilde{\omega}}
$$

is plotted in Fig.(1), from which it is clear that mean energy of the emitted particles are $\tilde{\omega} \approx a$. From (2.2) and Fig.(1), one can argue that, if the acceleration is such that

$$
a \geq \sum_{i} m_{q_{1}},
$$


the corresponding process (2.1) becomes energetically favored. In fact, more precise estimates reveal that the threshold is $a \geq \Delta m+\sum_{i} m_{q_{1}}$, where $\Delta m=m_{2}-m_{1}$. Hence, the neutral pion production (2.3) is expected do be favored for $a \geq m_{\pi} \approx 140 \mathrm{MeV}$. For the physical relevant case of protons in circular motion, one has also exactly the same threshold (2.8). For such case, the formulas for the neutral pion production (2.3) can be obtained from the previously one calculated in [6] for scalar emissions

$$
W_{s} \approx \frac{G_{\mathrm{eff}}^{(s) 2} a^{2}}{12 \pi} .
$$

We notice that, in order to obtain that the total power emission (2.9) from Eq. (4.7) of [6], one must perform the following modifications on the particle states: $\left|\pi^{+}\right\rangle \mapsto\left|\pi^{0}\right\rangle$ and $|n\rangle \mapsto|p\rangle$. These substitutions ensure the charge conservation and that the initial and final charged nucleon states are coupled in the same way to the magnetic field. In (2.9), $G_{\text {eff }}^{(s)}$ is related to the strong coupling constant $g$ by

$$
G_{\text {eff }}^{(s) 2}=\frac{g^{2}}{4 \pi} \approx 14
$$

The large difference between the strong and the electromagnetic coupling constants will imply a large difference also in the respective emitted powers. The comparison between (2.9) and the Larmor formula for the usual synchrotron radiation shows that $W_{s} / W_{\gamma}=g^{2} / 4 \pi \alpha \gg 1$, where $\alpha \approx$ $1 / 137$ is the electromagnetic coupling constant. Hence, if the proper acceleration of a proton reach the value $a \approx m_{\pi}$, the channel (2.3) is expect to dominate over the usual synchrotron radiation emission.

It must be emphasized that the formalism presented here cannot be applied when the charge currents corresponding to initial and final states are different. Neutral and charged particles follow distinct trajectories in the presence of an electromagnetic field. The related channel $p^{+} \rightarrow n \pi^{+}$for example, requires a different semi-classical formalism. The formalism and the respective calculations can be found in [8] (see also [9]).

\section{Applications}

Pulsar magnetospheres offer many interesting possibilities to test these results involving high energy accelerated particles[10, 11, 12]. Let us consider, for this purpose, the simplest polar cap models of pulsars $[13,14,15]$. For such models, a proton interacts with the dipolar magnetic field of the pulsar and is accelerated by an electric field parallel to the magnetic field lines. The transverse to the magnetic field component $p_{\perp}$ of the proton momentum vanish rapidly due to the usual synchrotron emission. The protons are accelerated along the magnetic field lines by the electric potential drop of the pulsar, producing photons by curvature radiation.

A particle following a circular trajectory of radius $R_{\mathrm{c}}$ has proper centripetal acceleration given by $a \approx \gamma^{2} / R_{\mathrm{c}}$. The curvature radius $R_{\mathrm{c}}$ of the magnetic field line of a pulsar is related to the radius $r_{\mathrm{S}}$ of the star and to the light cone radius, $R_{\mathrm{L}}=P / 2 \pi$ through $R_{\mathrm{c}}=4 / 3\left(r_{\mathrm{s}} R_{\mathrm{L}}\right)^{1 / 2}$, where $P$ is the star period. Then, one has

$$
a \approx \frac{\eta^{2} e^{2} \phi^{2}}{m^{2} R_{\mathrm{c}}} .
$$




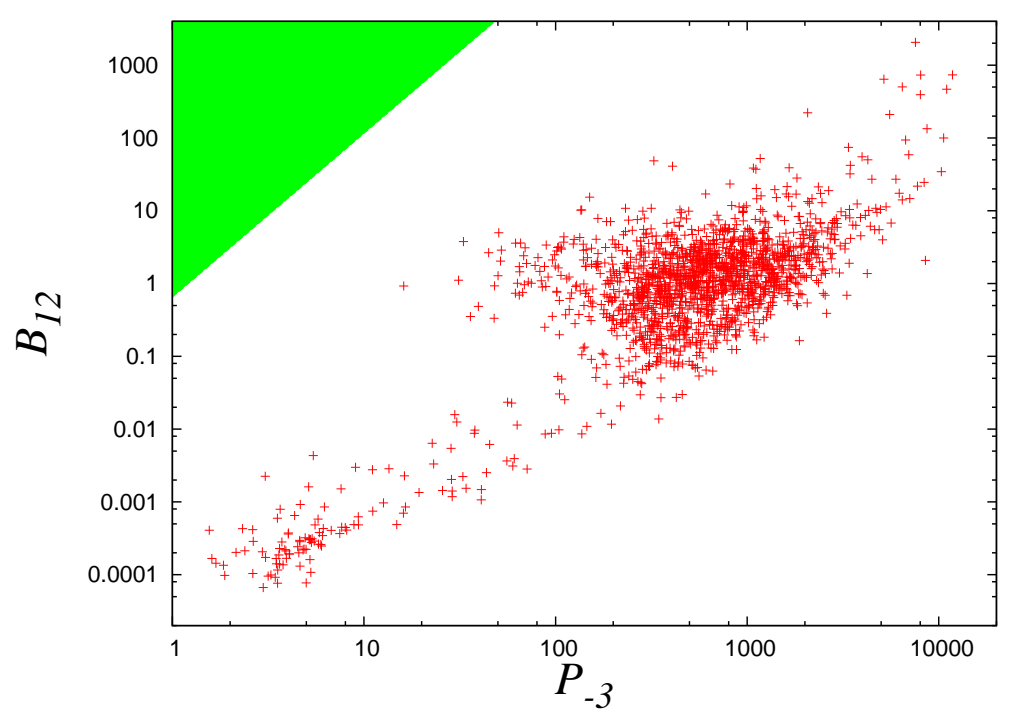

Figure 2: The values $\left(P_{-3}, B_{12}\right)$ for the 1700 objects in the ATNF Pulsar Catalogue[19] with known period $P=P_{-3} \times 10^{-3} \mathrm{~s}$ and surface magnetic field $B=B_{12} \times 10^{12} \mathrm{G}$. The shadowed region corresponds to the condition (3.3). All the pulsars in the ATNF catalogue are far below the pion production threshold.

The $\eta$ factor accounts for the efficiency of the polar cap acceleration mechanism $[16,17] \gamma=$ $\varepsilon / m=\eta e \phi / m$. Also,

$$
\phi=\left(\frac{2 \pi^{2} B r_{\mathrm{s}}^{3}}{P^{2}}\right) \approx 6.6 \times 10^{18} B_{12} P_{-3}^{-2}\left(\frac{r_{\mathrm{s}}}{10^{4} \mathrm{~m}}\right)^{3} \mathrm{~V},
$$

is the maximum available potential drop near the surface of the star [11, 18]. Taking for a typical young pulsar $r_{\mathrm{s}} \sim 10^{4} \mathrm{~m}$, one has from (3.1) and (2.8), the following condition on the star surface magnetic field

$$
B_{12} \geq 0.65 \times P_{-3}^{9 / 4}
$$

for the occurrence of pion emission with non negligible intensity. It is easily verified that even for $\eta=1$, the condition (3.3) is far above of the known pulsars of the ATNF Catalogue[19], see Fig. 2. In fact, a systematic search in the ATNF pulsar catalogue for strongly magnetized stars that could accelerate relativistic protons up to the curvature pion production threshold reveals that the best candidate is the $16 \mathrm{~ms}$ pulsar J0537-6910, but the corresponding characteristic parameter $\chi=a / m_{p}=10^{-6}$ is too small to give origin to observable signals [20] .

\section{Conclusions}

We have briefly reviewed the role played by the source proper acceleration in the semi-classical approximation for the process of massive particle emission. Unfortunately, we have from Eq.(3.3) and Fig. 2 that the necessary threshold for pion production by protons in circular motion is far above the typical acceleration experimented by protons in pulsar magnetospheres. Nevertheless, it would be also interesting to investigate, in this context, other radiative processes like photopion production and the inverse Compton scattering. 


\section{Acknowledgements}

The authors are grateful to A. T. Dias, C. O. Escobar, G. E. A. Matsas, R. Opher, S. Razzaque, and V. Pleitez for enlightening and helpful discussions.

\section{References}

[1] Ginzburg V L and Syrovatskii S I 1965 Ann. Rev. Astron. Astrophys. 3297

[2] Zharkov G F 1965 Sov. Phys. JETP 201525

[3] Erber T 1966 Rev. Mod. Phys. 38626

[4] Jackson J D 1999 Classical electrodynamics 3rd ed (New York, NY: Wiley)

[5] Vanzella D A and Matsas G E 2001 Phys. Rev. D63 014010

[6] Fregolente D, Matsas G E A and Vanzella D A T 2006 Phys. Rev. D74 045032

[7] Gradshteyn I S and Ryzhik I S 2004 Table of Integrals, Series, and Products (New York, NY: Academic Press)

[8] Herpay T and Patkós A 2008 J. Phys. G 35025201

[9] Fregolente D and Saa A 2008 Phys. Rev. D77 103010

[10] Berezinsky V, Dolgov A and Kachelrieß M 1995 Phys. Lett. B 351261

[11] Herpay T, Razzaque S, Patkós A and Mészáros P 2008 J. Cosm. Astropart. Phys. 825

[12] Tokuhisa A and Kajino T 1999 Astrophys. J. 525 L117

[13] Ruderman M A and Sutherland P G 1975 Astrophys. J. 19651

[14] Harding A K, Usov V V and Muslimov A G 2005 Astrophys. J. 622531

[15] Vietri M 2008 Foundations of High-Energy Astrophysics Theoretical Astrophysics (Chicago, IL: Chicago Univ. Press)

[16] Arons J 2003 Astrophys. J. 589871

[17] Zhang B, Dai Z G, Mészáros P, Waxman E and Harding A K 2003 Astrophys. J. 595346

[18] Harding A K and Muslimov A G 2001 Astrophys. J. 556987

[19] Manchester R N, Hobbs G B, Teoh A and Hobbs M 2005 Astron. J. 1291993 [arXiv:astro-ph/0412641].

[20] Fregolente D and Saa A 2011 Searching for curvature pion radiation from protons in strongly magnetized pulsars [arXiv:1101.1542] 